\title{
THEORETICAL SPECTRAL MODELS OF THE PLANET HD 209458b WITH A THERMAL INVERSION AND WATER EMISSION BANDS
}

\author{
A. Burrows, ${ }^{1}$ I. Hubeny, ${ }^{1}$ J. Budaj, ${ }^{1,2}$ H. A. Knutson, ${ }^{3}$ and D. Charbonneau ${ }^{3}$ \\ Received 2007 August 1; accepted 2007 August 28; published 2007 September 26
}

\begin{abstract}
We find that a theoretical fit to all the HD 209458b data at secondary eclipse requires that the day-side atmosphere of HD 209458b have a thermal inversion and a stratosphere. This inversion is caused by the capture of optical stellar flux by an absorber of uncertain origin that resides at altitude. One consequence of stratospheric heating and temperature inversion is the flipping of water absorption features into emission features from the near- to the mid-infrared, and we see evidence of such a water emission feature in the recent HD 209458b IRAC data of Knutson et al. In addition, an upper-atmosphere optical absorber may help explain both the weaker-thanexpected $\mathrm{Na} \mathrm{D}$ feature seen in transit and the fact that the transit radius at $24 \mu \mathrm{m}$ is smaller than the corresponding radius in the optical. Moreover, it may be a factor in why HD 209458b's optical transit radius is as large as it is. We speculate on the nature of this absorber and the planets whose atmospheres may, or may not, be affected by its presence.
\end{abstract}

Subject headings: planetary systems — planets and satellites: general — stars: individual (HD 209458)

\section{INTRODUCTION}

Of the more than 235 extrasolar giant planets (EGPs) discovered in the last 12 years, ${ }^{4} 22$ are transiting their primaries. The most thoroughly studied transiting EGP is HD 209458b (Henry et al. 2000; Charbonneau et al. 2000; Brown et al. 2001; Melo et al. 2006; Santos et al. 2004; Knutson et al. 2007b). For transiting EGPs, not only do we measure the planet's radius, but the $\sin i$ ambiguity of radial velocity studies is resolved to yield the planet's mass. Moreover, with precision photometry, the wavelength dependence of the transit radii can provide a measure of a planet's atmospheric composition (Burrows et al. 2000; Hubbard et al. 2001; Fortney et al. 2003; Barman 2007). In this way, sodium (Charbonneau et al. 2002) and water (Barman 2007) have been identified in HD 209458b and water has been identified in HD 189733b (Tinetti et al. 2007) and in TrES-1 (Burrows et al. 2005). Moreover, using the microsatellite MOST, high-precision optical photometry has constrained (perhaps, measured) HD 209458b's geometric albedo (Rowe et al. 2006, 2007). It is very low $(\sim 4.0 \% \pm 4.0 \%)$, in keeping with the predictions of Sudarsky et al. (2000) when the alkali metals, and not clouds, dominate absorption in the atmosphere and Rayleigh scattering dominates scattering. This makes HD 209458b darker in the optical than most of the objects of our solar system and also suggests that the associated contrast ratios are not optimal for characterizing EGPs.

However, for hot, close-in EGPs such as HD 209458b, the planet-star contrast ratios in the mid-infrared are much more favorable than in the optical (Burrows et al. 2004a, 2004b; Burrows 2005), often exceeding $10^{-3}$, and such contrasts are within reach of the infrared space telescope Spitzer (Werner \& Fanson 1995). Using its IRAC and MIPS cameras and the IRS spectrometer, one can now measure the summed light of the

\footnotetext{
${ }^{1}$ Department of Astronomy and Steward Observatory, University of Arizona, Tucson, AZ 85721; burrows@zenith.as.arizona.edu, hubeny@aegis .as.arizona.edu, budaj@as.arizona.edu.

${ }^{2}$ Astronomical Institute, Tatranska Lomnica, 05960 Slovak Republic.

${ }^{3}$ Harvard-Smithsonian Center for Astrophysics, 60 Garden Street, Cambridge, MA 02138; hknutson@cfa.harvard.edu, dcharbonneau@cfa.harvard.edu.

${ }^{4}$ See J. Schneider's Extrasolar Planet Encyclopedia at http://exoplanet.eu, the Geneva Search Program at http://exoplanets.eu, and the Carnegie/California compilation at http://exoplanets.org.
}

planet and the star in and out of secondary eclipse (when the star occults the planet) and, from the difference, determine the planet's spectrum at superior conjunction. This has led to a breakthrough in the study of exoplanets and a means to probe their chemistry and atmospheres. Hence, for the close-in EGPs in the near- to mid-infrared, and without the need to separately image planet and star, the direct detection of planetary atmospheres via low-resolution spectroscopy and precision IR photometry is now a reality (Charbonneau et al. 2005; Deming et al. 2005, 2006, 2007; Grillmair et al. 2007; Richardson et al. 2007; Harrington et al. 2006, 2007; Knutson et al. 2007a, 2007c; Cowan et al. 2007).

To date, secondary-eclipse fluxes in the IRAC and MIPS channels have been measured for five transiting EGPs (HD 189733b, TrES-1, HD 209458b, HD 149026b, and GJ 436b), but in this Letter we focus on the interpretation of the HD 209458 b data. For a more comprehensive paper on our numerical technique and theoretical fits to other secondary-eclipse data, as well as to phase light curve measurements, see Burrows et al. (2007a). For HD 209458b, not only is there a complete set of measurements at secondary eclipse using IRAC and MIPS, but using the IRS spectrometer, a low-resolution spectrum between $\sim 7.5$ and $\sim 15 \mu \mathrm{m}$ has now been obtained (Richardson et al. 2007; Swain et al. 2007).

Excitingly, the new IRAC data of Knutson et al. (2007a) trace out a positive bump in the $\sim 3.6-8 \mu \mathrm{m}$ spectral region, which we interpret via detailed modeling as a water emission feature. A water absorption feature was anticipated (Burrows et al. 2005, 2006; Fortney et al. 2005; Barman et al. 2005). Therefore, this is strong evidence for a thermal inversion in the atmosphere of HD 209458b which has flipped water absorption features into emission features. We now provide the comparison between theory and the observations and the resulting preliminary analysis.

\section{FIT TO THE HD 209458b PLANET/STAR FLUX RATIOS AT SECONDARY ECLIPSE}

Three models with an upper-atmosphere absorber in the optical (and with the resulting stratospheres) for the planet/star flux ratios of HD 209458b at secondary eclipse are portrayed in Figure 1. The purple, green, and red models are for redis- 


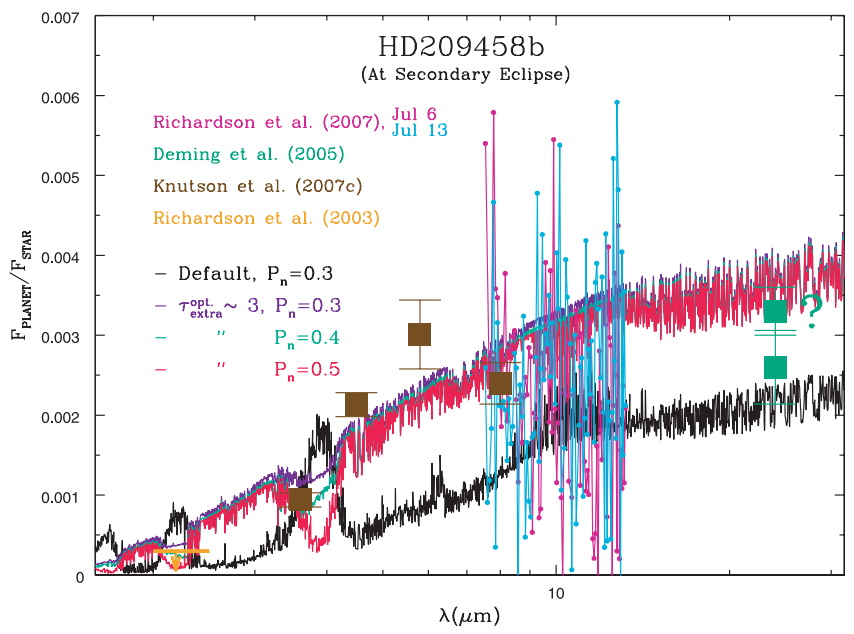

FIG. 1.-Planet-star flux ratios at secondary eclipse vs. wavelength for three models of the atmosphere of HD 209458b with inversions and for one model without an extra upper-atmosphere absorber of any kind (black; old default). The three models with stratospheres have different values of $P_{n}(=0.3$ [purple], 0.4 [green], and 0.5 [red]), but are otherwise the same. The old, default model has a $P_{n}$ of 0.3 . This figure demonstrates that models with an extra upper-atmosphere absorber in the optical and with $P_{n} \geq 0.35$ fit the data; the old, default model fits not at all. We have also calculated models (not shown) with equilibrium $\mathrm{TiO} / \mathrm{VO}$ in the upper atmosphere ( $\leq 30 \mathrm{mbars}$ ) (Burrows et al. 2006, 2007a), which are quite close to the corresponding models with an extra absorber. Superposed are the data in the $K$ band $(\sim 2.2 \mu \mathrm{m})$ from Richardson et al. (2003) (orange line and arrow), the four IRAC points from Knutson et al. (2007a) (brown square blocks), the IRS spectra from Richardson et al. (2007) (purple and aqua), and the MIPS data at $24 \mu \mathrm{m}$ from Deming et al. (2005) (green square block). Also included, with a question mark beside it, is a tentative update to this $24 \mu \mathrm{m}$ flux point, kindly provided by D. Deming (2007, private communication). If the flux at $24 \mu \mathrm{m}$ is indeed $\sim 0.0033 \pm$ 0.0003 , then our model(s) with inversions fit there as well. See the text for discussions.

tribution parameters ( $P_{n}$; Burrows et al. 2006) of 0.3, 0.4, and 0.5 , respectively, but are otherwise the same. For each model, an extra absorber of uncertain provenance is placed at altitude below pressures of 25 mbars. The total monochromatic optical depth of this layer is $\sim 3$. A new redistribution algorithm that introduces a heat sink on the day side between 0.01 and 0.1 bars, and a corresponding source on the night side, is employed. This algorithm is explained in Burrows et al. (2007a). Following the suggestion of Hubeny et al. (2003) in their atmosphere bifurcation study, we have also calculated a model suite (not shown) with equilibrium $\mathrm{TiO}$ and $\mathrm{VO}$ in the stratosphere. With a total Planck-mean optical depth of TiO/VO below $~ 30$ mbars of $\sim 3.1$, these models are quite similar to those with inversions depicted in Figure 1 with the same $P_{n}$ values. Figure 1 also provides a representative default model (black) without a stratospheric absorber, but with $P_{n}=0.3$, along with all the relevant HD 209458b measurements to date. The default model represents the previous predictions for atmospheres with monotonically decreasing temperatures and no inversions, although the new redistribution algorithm alluded to above was employed.

The most salient observational constraints for our current purposes are the geometric albedo in the optical from MOST (Rowe et al. 2006, 2007), a $K$-band upper limit using IRTF SpeX from Richardson et al. (2003), a MIPS $24 \mu \mathrm{m}$ photometric point from Deming et al. (2005), a low-resolution Spitzer IRS spectrum from Richardson et al. (2007), and, most importantly, photometric points in IRAC channels 1-4 from Knutson et al. (2007a). Richardson et al. (2007) suggest that there is evidence in the IRS data for two spectral features: one near $7.78 \mu \mathrm{m}$ and one near $9.67 \mu \mathrm{m}$. However, we think the data are too noisy to draw this conclusion and await the next generation of observations to test it. Moreover, we note that Richardson et al. (2007) normalize their data to the Knutson et al. (2007a) IRAC 4 point, but that due to the noisiness of the IRS data near $8 \mu \mathrm{m}$ this normalization deserves a second look.

The $1 \sigma$ optical albedo limit from Rowe et al. (2007) is $\sim 8.0 \%$, a very low number. For comparison, the geometric albedo for Jupiter is $\sim 40 \%$. However, such a low number was predicted due to the prominence in the optical of broadband absorptions by the alkali metals sodium and potassium in the hot atmospheres of irradiated EGPs (Sudarsky et al. 2000; their "Class IV"). Alkali metals are not found in Jupiter's atmosphere. The associated planet-star flux ratios are $\sim 10^{-5}$ to $10^{-6}$. A low albedo due to absorption by the $\mathrm{Na} \mathrm{D}$ and $\mathrm{K}$ I resonance lines is consistent with the identification of sodium in the atmosphere of HD 209458b using HST STIS transit spectroscopy (Charbonneau et al. 2002), although the magnitude of the sodium feature has yet to be fully explained. Both these data sets suggest that any clouds that might reside in the atmosphere of HD 209458b are thin or mostly absorbing. A thick scattering cloud layer would reflect light rather efficiently and lead to a high albedo, which is not seen. The extra absorber we introduce could in fact be a cloud, but in that case it must be comprised of particles with a low scattering albedo. This would seem to eliminate forsterite and enstatite.

As Figure 1 demonstrates, the low upper limit of Richardson et al. (2003) in the $K$ band that was problematic in the previous theory (Burrows et al. 2005; Fortney et al. 2005; Barman et al. 2005) is comfortably consistent with the models with an extra upper-atmosphere absorber in the optical, particularly for higher values of $P_{n}$. Moreover, the peak near the IRAC 1 channel $(\sim 3.6 \mu \mathrm{m})$ in the previous model without an inversion (Burrows et al. 2006) is reversed with the extra absorber into a deficit that fits the Knutson et al. (2007a) point. Importantly, the theory without an extra absorber at altitude predicts that the planetstar flux ratio in the IRAC 2 channel should be lower than the corresponding ratio at IRAC 1 . However, with the extra absorber the relative strengths in these bands are reversed, just as are the Knutson et al. (2007a) points for HD 209458b. This reversal is a clear signature of a thermal inversion in the lowpressure regions of the atmosphere. Figure 2 depicts the corresponding temperature-pressure profiles and the thermal inversion at low pressures introduced by the presence of an extra absorber in the optical. It also indicates the approximate positions of the effective photospheres for photons in the IRAC and MIPS channels. The fact that in the new models the IRAC 1 photosphere is cooler than the IRAC 2 photosphere is a key to the observed behavior of the Knutson et al. (2007a) data.

Figure 1 indicates that the models with a stratosphere fit the IRAC 1, 2, and 4 flux points quite well. However, the relative height of the IRAC 3 point near $5.8 \mu \mathrm{m}$ is difficult to fit, while maintaining the good fits at the other IRAC points and consistency with the Richardson et al. (2003) limit. As Figure 2 shows, the positions of the IRAC 3 and IRAC 4 photospheres are generally close to one another. This makes it difficult to have effective "brightness" temperatures that are as different as are implied by the IRAC data. Nevertheless, IRAC 2, 3, and 4 together trace out a peak, whereas in the default theory an absorption trough was expected. Note in Figure 1 the significant difference in the planet-star flux ratio at these wavelengths between the old default model and the new models with a stratospheric absorber. This is the spectral region of a strong 


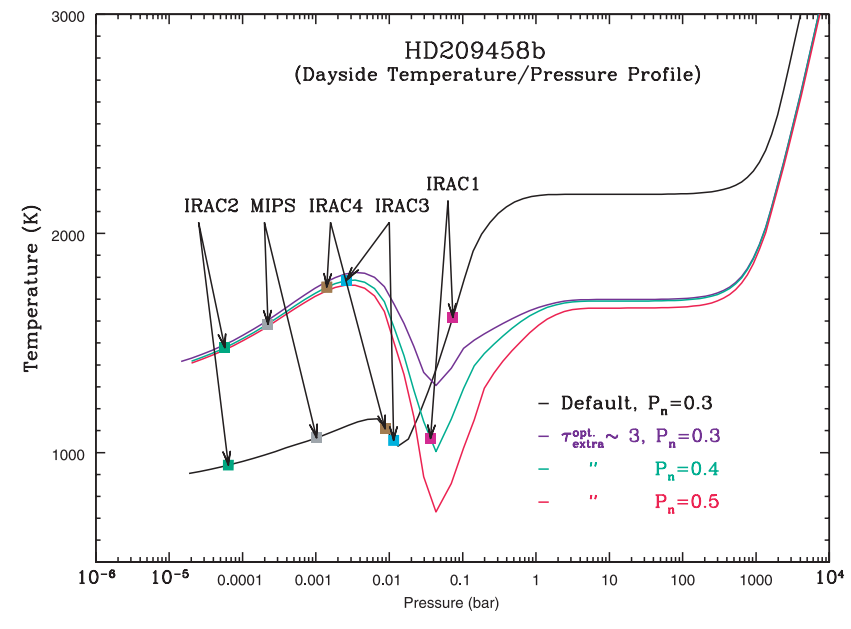

FIG. 2.-Day side temperature (K)-pressure (bars) profiles for the four models for HD 209458b depicted in Fig. 1. For the old, default model and the new $P_{n}=0.4$ model, we indicate the positions of the "photospheres" for the IRAC and MIPS $24 \mu \mathrm{m}$ bands, defined as the corresponding $\tau=2 / 3$ surfaces. Most of these mid-infrared photospheres are at altitude, where an extra absorber can have a significant effect. The photospheres for the near-IR $J, H$, and $K$ bands (as well as the IRAC 1 band) are deeper in. A comparison of the green and the black curves demonstrates that both cooling by heat redistribution $\left(P_{n} \gtrsim 0.3\right)$ and heating by absorption in the upper atmosphere together are necessary to invert the IRAC1/IRAC2 flux ratio. All three inversion models experience some cooling at moderate pressures due to a sink imposed to mimic heat redistribution, but this process alone is insufficient to elevate the temperatures of the upper atmosphere to values necessary to fit the Knutson et al. (2007a) IRAC data for HD 209458b. An extra absorber in the optical and at low pressures is called for. See text for a discussion.

rovibrational band of water, and a comparison between our new HD 209458b models and the data indicates that this feature is in emission.

The IRS data of Richardson et al. (2007) are normalized to the Knutson et al. (2007a) IRAC 4 point, so the good fit there is not independent. Nevertheless, the IRS data have a flattish slope between $\sim 7.5$ and $\sim 14 \mu \mathrm{m}$ that is mildly inconsistent with the slight rise of our new models. In addition, the $24 \mu \mathrm{m}$ MIPS point obtained by Deming et al. (2005) is lower than these models. However, the flux at this point is being reevaluated (D. Deming 2007, private communication). If the new value at $24 \mu \mathrm{m}$ is, as suggested, $\sim 0.0033 \pm 0.0003$, then our new model(s) with inversions fit here as well (see Fig. 1). ${ }^{5} \mathrm{Be}$ that as it may, the good qualitative and quantitative fits for the $K$-band limit, three of the four IRAC channels, and the MOST albedo limit (not shown) lend credence to our overall model and conclusions. Concerning the day-side atmosphere of HD 209458 b, there is an extra absorber in the optical at altitude, there is a pronounced thermal inversion (see Fig. 2), and water is seen in emission.

\section{DISCUSSION}

We find that a consistent fit to all the HD 209458b data at secondary eclipse requires that the atmosphere of HD 209458b have a thermal inversion at altitude and a stratosphere. This inversion is caused by the capture of incident optical stellar flux by an extra absorber of currently uncertain origin that resides at low pressures. The IRAC data of Knutson et al. (2007a) cannot be fit by the effects of day-side heat redistri-

\footnotetext{
${ }^{5}$ Note, however, that the actual updated number has yet to be determined and published.
}

bution cooling alone. A consequence of stratospheric heating and temperature inversion is the flipping of water absorption features into emission features from the near- to the mid-infrared (Hubeny et al. 2003; Burrows et al. 2006), and this seems to explain all the current HD 209458b data. Hence, contrary to the interpretation of Richardson et al. (2007), the flatness or slight rise of their IRS spectrum near $\sim 7.8 \mu \mathrm{m}$ (Fig. 1) in fact supports the presence of abundant atmospheric water, because this region is at the edge of a strong water band in emission. Our inference of the presence of water in abundance in the atmosphere of HD 209458b is also consistent with the conclusion of Barman (2007) using transmission spectroscopy.

If the extra absorber is in the gas phase, and there is no cloud, then our new models are easily consistent with the low albedo derived by Rowe et al. (2006, 2007). If the extra absorber is a cloud, the cloud particles must have a low scattering albedo and cannot be very reflecting. This would seem to rule out forsterite or enstatite clouds, but does not necessarily rule out iron clouds. As shown by Hubeny et al. (2003) and Burrows et al. (2006), strongly irradiated atmospheres can experience a solution bifurcation to an atmosphere with an inversion for which the water features are flipped from absorption to emission. In those papers, the absorber was gas-phase $\mathrm{TiO} / \mathrm{VO}$, which in equilibrium can exist at low pressures at altitude and not just at high temperatures at depth. If the extra absorber were $\mathrm{TiO} / \mathrm{VO}$, there would be fewer free parameters, but it was thought that the "cold trap" effect would quickly deplete the upper atmosphere of $\mathrm{TiO} / \mathrm{VO}$ and ensure the default atmospheric solution without an inversion. However, this has not been proven, particularly when atmospheric mass loss is ongoing, as we know to be the case for HD 209458b (VidalMadjar et al. 2003, 2004). The same arguments hold for iron clouds, although since absorption by iron particles is not restricted to the optical, this solution is suboptimal.

One can also speculate that the severe irradiation regime of some close-in EGPs might create nonequilibrium compounds through photolysis, such as the tholins or polyacetylenes discussed in the more benign contexts of solar system bodies, that could serve as the extra absorber we deduce exists in the atmosphere of HD 209458b. Clearly, what the high-altitude absorber actually is remains to be seen, but one can speculate that its presence is more likely in the atmospheres of the most strongly irradiated EGPs. What "most strongly irradiated" actually means is not yet clear, but the flux at the substellar point on HD $209458 \mathrm{~b}$ is $\sim 10^{9} \mathrm{ergs} \mathrm{cm}^{-2} \mathrm{~s}^{-1}$. The corresponding numbers for OGLE-TR-10b, OGLE-TR-56b, OGLE-TR-132b, TrES-2, TrES-3, WASP-1b, XO-3b, HAT-P-1b, and HD 149026b are higher (Burrows et al. 2007b). The corresponding number for TrES-1 is lower $\left(\sim 0.43 \times 10^{9} \mathrm{ergs} \mathrm{cm}^{-2} \mathrm{~s}^{-1}\right)$, and this planet shows good evidence for water in absorption (Burrows et al. 2005). In addition, the IRS spectrum of HD 189733b of Grillmair et al. (2007) seems consistent with a more canonical water absorption feature shortward of $\sim 8 \mu \mathrm{m}$. Its substellar flux is $\sim 0.47 \times 10^{9} \mathrm{ergs} \mathrm{cm}^{-2} \mathrm{~s}^{-1}$. So, if stellar flux at the planet is an indicator, we may have a handle on which planets reside in the transition region between manifesting water absorption or emission features (and inversions), and where the relative IRAC 1/IRAC 2 strengths start to flip. Given their substellar fluxes, HD 189733b, XO-1b, XO-2b, and/or WASP-2b may be links. Although we find a weak dependence on metallicity, nonequilibrium chemistry and cloud formation may have stronger dependences. Therefore, there are still numerous parameters to address.

As Figure 1 suggests, the planet/star ratios shortward of $\sim 4 \mu \mathrm{m}$ 
are more sensitive to the heat redistribution parameter $P_{n}$ than the corresponding ratios longward of $\sim 8 \mu \mathrm{m}$. This suggests a shorter wavelength strategy for designing close-in EGP climate diagnostics. In addition, models with inversions significantly boost the mid-infrared fluxes longward of $\sim 15 \mu \mathrm{m}$. This boost is not the only signature of models with day-side stratospheres. Since the night-side flux at the same $P_{n}$ is unlikely to be much altered by the fact of day-side inversion, if the day side has such an inversion due to enhanced optical absorption at altitude, the day-night contrasts measured during an orbital traverse by a close-in EGP will be larger than expected for a given $P_{n}$. This possibility is relevant when interpreting the $24 \mu \mathrm{m}$ light curve of $v$ And $b$ (Harrington et al. 2006). Perhaps, the large daynight contrast seen in this case does not require a small $P_{n}$.

An upper-atmosphere absorber in the optical may simultaneously explain the weaker-than-expected $\mathrm{Na} \mathrm{D}$ feature seen in transit by Charbonneau et al. (2002) (Fortney et al. 2003) and the fact that the transit radius measured by Richardson et al. (2006) at $24 \mu \mathrm{m}\left(1.26 \pm 0.08 R_{\mathrm{J}}\right)$ is smaller than the corresponding radius in the optical $\left(1.32 \pm 0.025 R_{\mathrm{J}}\right.$; Knutson et al. 2007b). In addition, it may help explain why HD 209458b's optical transit radius is as large as it is. Finally, the extra absorber may be related to the enhanced opacities for close-in EGPs discussed in the context of the EGP radius models of Burrows et al. (2007b). Whatever their actual roles, anomalous optical absorption at altitude and thermal inversions are now insinuating themselves as exciting new components of EGP theory.

We thank Drake Deming, Bill Hubbard, Maki Hattori, Mike Cushing, and Drew Milsom for helpful discussions. This study was supported in part by NASA grants NNG04GL22G and NNX07AG80G and through the NASA Astrobiology Institute under cooperative agreement CAN-02-OSS-02 issued through the Office of Space Science.

\section{REFERENCES}

Barman, T. 2007, ApJ, 661, L191

Barman, T. S., Hauschildt, P. H., \& Allard, F. 2005, ApJ, 632, 1132

Brown, T. M., Charbonneau, D., Gilliland, R. L., Noyes, R. W., \& Burrows, A. 2001, ApJ, 552, 699

Burrows, A. 2005, Nature, 433, 261

Burrows, A. Guillot, T., Hubbard, W. B., Marley, M. S., Saumon, D., Lunine, J. I., \& Sudarsky, D. 2000, ApJ, 534, L97

Burrows, A., Hubeny, I., \& Budaj, J. 2007a, ApJ, submitted (arXiv:0709.4080)

Burrows, A., Hubeny, I., Budaj, J., \& Hubbard, W. B. 2007b, ApJ, 661, 502

Burrows, A., Hubeny, I., \& Sudarsky, D. 2005, ApJ, 625, L135

Burrows, A., Sudarsky, D., \& Hubeny, I. 2004a, in AIP Conf. Proc. 723, The Search for Other Worlds, ed. S. Holt \& D. Deming (New York: AIP), 143 2004b, ApJ, 609, 407 2006, ApJ, 650, 1140

Charbonneau, D., Brown, T. M., Latham, D. W., \& Mayor, M. 2000, ApJ, 529, L45

Charbonneau, D., Brown, T. M., Noyes, R. W., \& Gilliland, R. L. 2002, ApJ, 568,377

Charbonneau, D., et al. 2005, ApJ, 626, 523

Cowan, N.B., Agol, E., \& Charbonneau, D. 2007, MNRAS, 379, 641

Deming, D., Harrington, J., Laughlin, G., Seager, S., Navarro, S. B., Bowman, W. C., \& Horning, K. 2007, ApJ, 667, L199

Deming, D., Harrington, J., Seager, S., \& Richardson, L. R. 2006, ApJ, 644, 560

Deming, D., Seager, S., Richardson, L. J., \& Harrington, J. 2005, Nature, 434, 740

Fortney, J. J., Marley, M. S., Lodders, K., Saumon, D., \& Freedman, R. S. 2005, ApJ, 627, L69

Fortney, J. J., Sudarsky, D., Hubeny, I., Cooper, C. S., Hubbard, W. B., Burrows, A., \& Lunine, J. I. 2003, ApJ, 589, 615

Grillmair, C. J., Charbonneau, D., Burrows, A., Armus, L., Stauffer, J., Meadows, V., Van Cleve, J., \& Levine, D. 2007, ApJ, 658, L115
Harrington, J., Hansen, B., Luszcz, S., Seager, S., Deming, D., Menou, K., Cho, J., \& Richardson, L. 2006, Science, 314, 623

Harrington, J., Luszcz, S., Seager, S., Deming, D., \& Richardson, L. J. 2007, Nature, 447, 691

Henry, G., Marcy, G. W., Butler, R. P., \& Vogt, S. S. 2000, ApJ, 529, L41

Hubbard, W. B., Fortney, J. J., Lunine, J. I., Burrows, A., Sudarsky, D., \& Pinto, P. 2001, ApJ, 560, 413

Hubeny, I., Burrows, A., \& Sudarsky, D. 2003, ApJ, 594, 1011

Knutson, H. A., Charbonneau, D., Allen, L. E., Torres, G., Burrows, A., \& Megeath, S. T. 2007a, ApJ, submitted

Knutson, H. A., Charbonneau, D., Noyes, R. W., Brown, T. M., \& Gilliland, R. L. 2007b, ApJ, 655, 564

Knutson, H. A., et al. 2007c, Nature, 447, 183

Melo, C., et al. 2006, A\&A, 460, 251

Richardson, L. J., Deming, D., Horning, K., Seager, S., \& Harrington, J. 2007, Nature, 445, 892

Richardson, L. J., Deming, D., \& Seager, S. 2003, ApJ, 597, 581

Richardson, L. J., Harrington, J., Seager, S., \& Deming, D. 2006, ApJ, 649, 1043

Rowe, J. F., et al. 2006, ApJ, 646, 1241 2007, ApJ, submitted

Santos, N. C., Israelian, G., \& Mayor, M. 2004, A\&A, 415, 1153

Sudarsky, D., Burrows, A., \& Pinto, P. 2000, ApJ, 538, 885

Swain, M., Bouwman, J., Akeson, R., Lawler, S., \& Beichman, C. 2007, ApJL, submitted (astro-ph/0702593)

Tinetti, G., et al. 2007, Nature, 448, 169

Vidal-Madjar, A., Lecavelier des Etangs, A., Désert, J.-M., Ballester, G. E., Ferlet, R., Hébrand, G., \& Mayor, M. 2003, Nature, 422, 143

Vidal-Madjar, A., et al. 2004, ApJ, 604, L69

Werner, M. W., \& Fanson, J. L. 1995, Proc. SPIE, 2475, 418 